\title{
Phase evolution and electrical behaviour of samarium-substituted bismuth ferrite ceramics
}

Chuying Yu ${ }^{a}$, Giuseppe Viola ${ }^{\text {a,b }}$, Dou Zhang ${ }^{c, *}$, Kechao Zhou $^{c}$, Vladimir Koval ${ }^{d}$, Amit Mahajan ${ }^{a}$, Rory M. Wilson ${ }^{a}$, Nadezda V. Tarakina a ${ }^{\text {, Isaac Abrahams }}{ }^{\text {e }}$ and Haixue Yan ${ }^{\text {a,* }}$

a School of Engineering and Materials Sciences, Queen Mary University of London, Mile End Road London E1 4NS, UK.

${ }^{b}$ Department of Applied Science and Technology, Institute of Materials Physics and Engineering, Corso Ducadegli Abruzzi 24, 10129 Torino, Italy

' State Key Laboratory of Powder Metallurgy, Central South University, Changsha 410083, China

d Institute of Materials Research, Slovak Academy of Sciences, Watsonova 47, 04001 Kosice, Slovakia.

e School of Biological and Chemical Sciences, Queen Mary University of London, Mile End Road London E1 4NS, UK.

${ }^{*}$ Corresponding Authors:

D. Zhang, email: dzhang@csu.edu.cn

H. X. Yan, email: $\underline{\text { h.x.yan@qmul.ac.uk }}$

Key words: antiferroelectric; $\mathrm{Sm}-\mathrm{BiFeO}_{3}$; diffraction; dielectric 


\begin{abstract}
$\mathrm{Bi}_{1-x} \mathrm{Sm}_{x} \mathrm{FeO}_{3}(x=0.15-0.18)$ ceramics with high density were produced using spark plasma sintering. The effects of composition, synthesis conditions and temperature on the phase evolution were studied, using XRD, TEM and dielectric spectroscopy. The coexistence of the ferroelectric R3c, antiferroelectric Pnam and paraelectric Pnma phases was revealed, with relative phase fractions affected by both calcination conditions and Sm concentration. Experiments on powdered samples calcined at different temperatures up to $950{ }^{\circ} \mathrm{C}$ suggest higher calcination temperatures promote Sm diffusion, allowing samples to reach compositional homogeneity. The structural transitions from the Pnam and $R 3 c$ phases to the Pnma phase were comprehensively investigated, with phase transition temperatures clearly identified. The dielectric permittivity, electrical resistivity and breakdown strength were increased upon Sm-substitution, while ferroelectric switching was suppressed. The polarizationelectric field loop became increasingly narrow with increasing Sm-content, but double hysteresis loops, which may reflect a reversible antiferroelectric to ferroelectric transformation, were not observed.
\end{abstract}




\section{Introduction}

$\mathrm{BiFeO}_{3}$ (BFO) is a room-temperature multiferroic material with a rhombohedrally distorted $R 3 c$ perovskite structure [1]. It shows a high ferroelectric (FE) polarization of ca. 90$100 \mu \mathrm{C} \mathrm{cm}{ }^{-2}$ along the [111] direction, with a ferroelectric Curie point of $825{ }^{\circ} \mathrm{C}$ and an antiferromagnetic Néel temperature of $370{ }^{\circ} \mathrm{C}[1,2]$. However, the synthesis of pure BFO is difficult due to the narrow temperature window of compound formation, with secondary phases such as $\mathrm{Bi}_{2} \mathrm{Fe}_{4} \mathrm{O}_{9}$ and $\mathrm{Bi}_{25} \mathrm{FeO}_{40}$ often formed $[3,4]$. Additionally, the $\mathrm{BFO}$ system always exhibits significant electrical conductivity, due to the reduction of $\mathrm{Fe}^{3+}$ to $\mathrm{Fe}^{2+}$ and evaporation of volatile bismuth oxide [5]. It is reported that A-site substitution by rare-earth cations could compensate for the volatilization of bismuth oxide and suppress the formation of oxygen vacancies to reduce the electrical conductivity [6, 7]. Meanwhile, rare-earth substitution can enhance the dielectric and electromechanical properties [8-10], making the material a potential candidate for advanced piezoelectric, ferroelectric and multiferroic applications $[1,11,12]$.

A large number of studies have focused on the structural evolution in the phase diagrams of binary $\mathrm{BiFeO}_{3}-\mathrm{REFeO}_{3}$ systems (where $\mathrm{RE}=\mathrm{La}, \mathrm{Sm}, \mathrm{Nd}$ etc.) [13-18]. The isovalent substitution of bismuth by rare-earth elements leads to a structure transformation from the rhombohedral FE $R 3 C$ phase to an orthorhombic paraelectric Pnma phase, with the formation of a morphotropic phase boundary (MPB) $[7,16,19]$. An orthorhombic antiferroelectric (AFE) Pbam phase is reported to be formed in rare-earth-substituted BFO across the MPB region, where it coexists with the $R 3 C$ and Pnma phases $[8,20]$. However, there is an ongoing debate on the details of the structure-composition relationships in $\mathrm{BiFeO}_{3}-\mathrm{REFeO}_{3}$ systems $[9,13,21]$. 
In thin films, Kan et al. found that the structural behaviour of $\mathrm{BiFeO}_{3}-\mathrm{REFeO}_{3}$ systems is dictated by the average ionic radius of the A-site cations [21, 22]. However, this relationship is less clear in bulk ceramics, with different rare-earth systems exhibiting different phase behaviour irrespective of the average size of the A-site cation. This is further complicated by the effect of synthesis conditions and the level of substitution, which have also been shown to influence the phase distribution $[15,23]$.

The origin of the double polarization-electric (P-E) hysteresis loops observed in thin films is also controversial. It was initially considered as a result of an electric field-induced AFE to FE phase transition $[8,20]$. However, Kan et al. suggested that the double hysteresis loops were linked to an electric field-induced paraelectric (PE) to FE phase transition [21]. Using first principles calculations, these authors found that the energy of the PE Pnma phase was closer to that of the ground state $R 3 c$ phase, compared to that of the AFE Pbam phase [21]. Thus, it is easier for the Pnma phase rather than the Pbam phase to transform into the $R 3 c$ phase under an external electric field. To date, it is still difficult to obtain saturated polarization-electric field (P-E) hysteresis loops in rare-earth doped $\mathrm{BiFeO}_{3}$ ceramic samples, due to their low breakdown field and high leakage current [24-27]. Double hysteresis loops have never been observed in $\mathrm{BiFeO}_{3}$-based ceramic samples.

The focus of the current work is on Sm-substituted $\mathrm{BiFeO}_{3}$, where double P-E loops have been observed in thin films. It is agreed that $\mathrm{Bi}_{1-x} \mathrm{Sm}_{x} \mathrm{FeO}_{3}$ exhibits an $R 3 c$ structure at $x<0.1[16$, $28,29]$. However, the structure of compositions at and above $x=0.1$ differs between thin films and ceramics. The MPB region is suggested to be located at $x=0.14$ in $\mathrm{Bi}_{1-x} \mathrm{Sm}_{x} \mathrm{FeO}_{3}$ thin films [8]. Substitution by samarium in $\mathrm{Bi}_{1-x} \mathrm{Sm}_{x} \mathrm{FeO}_{3}$ (at $x \geq 0.1$ ) thin films destabilizes the long-range 
FE state to form localized AFE clusters within the FE matrix in the compositional range $0.10 \leq x \leq$ 0.14, with a Pnma phase observed in compositions with $x>0.14$ [30]. In bulk samples, Khomchenko et al. reported that $\mathrm{Bi}_{0.85} \mathrm{Sm}_{0.15} \mathrm{FeO}_{3}$ was a mixture of Pbam and Pnma phases, while a pure Pnma structure was found at $x=0.2$ [28]. Troyanchuk et al. reported that $\mathrm{Bi}_{1}$ ${ }_{x} \mathrm{Sm}_{x} \mathrm{FeO}_{3}$ ceramics consisted of a mixture of polar and antipolar phases, in the range $x=0.11$ 0.13 , while the antipolar phase was dominant at $x=0.13$ [29]. In particular, the antipolar phase was reported to have Pnam symmetry $\left(\sqrt{ } 2 a_{c} \times 2 \sqrt{ } 2 a_{c} \times 4 a_{c}\right.$, where $a_{c}$ is the pseudo-cubic perovskite cell dimension), with double the unit cell volume of the antipolar phase reported in other studies ( $\sqrt{ } 2 a_{\mathrm{c}} \times 2 \sqrt{ } 2 a_{\mathrm{c}} \times 2 a_{\mathrm{c}}$ in space group Pbam) [13]. The pure antipolar state was only obtained at $x=0.14$, while traces of the Pnma phase were detected at $x=0.15$ and the pure Pnma phase was obtained at $x=0.18$ [29].

The calcination temperature has also been found to influence the phase distribution in the $\mathrm{Bi}_{1-x} \mathrm{Sm}_{x} \mathrm{FeO}_{3}$ system $[23,31]$. Khomchenko et al. found that a low calcination temperature of $850{ }^{\circ} \mathrm{C}$ led to the coexistence of the Pbam, R3c and Pnma phases in the $x=0.15$ ceramic [23]. The phase separation was attributed to an inhomogeneous distribution of $\mathrm{Bi}^{3+}$ and $\mathrm{Sm}^{3+}$ cations caused by the low calcination temperature. The $R 3 c$ phase was absent after calcining at a higher temperature of $950{ }^{\circ} \mathrm{C}$. Even though preliminary phase diagrams of rare-earth doped $\mathrm{BiFeO}_{3}$ have been proposed, details of the transitions from the Pbam and $R 3 C$ phases to the high temperature Pnma phase remain vague. The identification of the characteristic temperatures associated with these transitions is complicated by the coexistence of several phases in the MPB region $[13,21]$. 
In this work, the phase evolution in $\mathrm{Bi}_{1-x} \mathrm{Sm}_{x} \mathrm{FeO}_{3}$ ceramics has been studied, including the effect of composition and temperature. X-ray diffraction and transmission electron microscopy were carried out to investigate the presence of an antiferroelectric structure. The temperature dependence of the X-ray diffraction patterns and dielectric properties were used to identify the temperatures associated with the different phase transitions. Polarization-electric field measurements were performed, searching for the possible presence of double hysteresis which could be related to an antiferroelectric to ferroelectric field-induced transition.

\section{Experimental Methods}

\subsection{Preparations}

The $\mathrm{Bi}_{1-x} \mathrm{Sm}_{x} \mathrm{FeO}_{3}$ compositions $(0.15 \leq x \leq 0.18)$ were selected as they are expected to show an MPB region, characterized by the possible presence of an AFE structure [8]. The starting materials $\mathrm{Bi}_{2} \mathrm{O}_{3}$ (Alfa Aesar, UK, 99.975\%), $\mathrm{Sm}_{2} \mathrm{O}_{3}$ (Alfa Aesar, UK, 99.9\%) and $\mathrm{Fe}_{2} \mathrm{O}_{3}$ (Alfa Aesar, UK, 99.9\%) were mixed and milled in ethanol using a planetary ball mill (Nanjing machine factory, China) for $4 \mathrm{~h}$. The dried powders were calcined at selected temperatures ( 810 ${ }^{\circ} \mathrm{C}, 830{ }^{\circ} \mathrm{C}, 850{ }^{\circ} \mathrm{C}, 910{ }^{\circ} \mathrm{C}$ or $950{ }^{\circ} \mathrm{C}$ ) for $4 \mathrm{~h}$ to identify the optimum calcination conditions. In each case, the synthesized powder was further milled in ethanol for $4 \mathrm{~h}$ to obtain fine homogenous particles. The $\mathrm{Bi}_{1-x} \mathrm{Sm}_{x} \mathrm{FeO}_{3}$ ceramics $(x=0.150,0.160,0.165$, denoted as BSF15, BSF16 and BSF16.5, respectively) were sintered at $800^{\circ} \mathrm{C}$ from powder calcined at $910^{\circ} \mathrm{C}$, using spark plasma sintering (SPS) (HPD-25/1 FCT systeme $\mathrm{GmbH}$ ) at a pressure of $80 \mathrm{MPa}$, with a heating rate of $100{ }^{\circ} \mathrm{C} \mathrm{min}-1$ and a dwell time of $5 \mathrm{~min}$. The $\mathrm{Bi}_{0.82} \mathrm{Sm}_{0.18} \mathrm{FeO}_{3}$ (denoted as BSF18) 
ceramic was sintered at $800{ }^{\circ} \mathrm{C}$ from powder calcined at $950{ }^{\circ} \mathrm{C}$ using the same sintering procedures. The SPS sintered samples were subsequently annealed in a muffle furnace (Carbolite HTF 1800, UK) at $700{ }^{\circ} \mathrm{C}$ in air for $15 \mathrm{~h}$ to remove the diffused carbon from the graphite during the SPS processing $[32,33]$.

\subsection{Structure analysis}

The structures of the calcined powders and sintered ceramics were analysed by X-ray powder diffraction (XRD, X'pert Pro, PANalytical, Almelo, The Netherlands) at room temperature over the $2 \theta$ range $5^{\circ}$ to $120^{\circ}$, in steps of $0.0167^{\circ}$, with an effective count rate of $200 \mathrm{~s}$ per step, using $\mathrm{Ni}$ filtered $\mathrm{Cu}-\mathrm{K} \alpha(1.5418 \AA ̊ \AA)$ radiation. Elevated temperature measurements were carried out on BSF15 and BSF18 calcined powders using an Anton-Paar HTK-16 high-temperature camera. The data were collected at $25{ }^{\circ} \mathrm{C}$ and at $50{ }^{\circ} \mathrm{C}$ intervals from $100{ }^{\circ} \mathrm{C}$ to $950{ }^{\circ} \mathrm{C}$. The sample was held at each temperature for ca. 45 min during data collection. Diffraction patterns were acquired over the $2 \theta$ range $5^{\circ}$ to $120^{\circ}$, in steps of $0.0167^{\circ}$, with an effective count rate of $50 \mathrm{~s}$ per step. The temperature calibration was carried out by measuring the phase transition of $\mathrm{KNO}_{3}$ and melting points of high purity $\mathrm{KNO}_{3}, \mathrm{KI}, \mathrm{NaCl}$ and $\mathrm{K}_{2} \mathrm{SO}_{4}$ standards. All the XRD patterns were modelled by the Rietveld method using the General Structure Analysis System (GSAS) software [34]. The initial structural models were based on those reported by Khomchenko et al. for the AFE phase with space group Pnam and the PE phase with space group Pnma [35] and by Moreau et al. for the FE phase with space group $R 3 c$ [36]. The microstructure was studied using scanning electron microscopy (SEM, FEI Inspect F). 
Selected area electron diffraction (SAED) patterns were recorded on BSF15 calcined powder using a JEOL 2010 transmission electron microscope. For this measurement, the powder was crushed and dispersed in ethanol and a drop of this suspension was placed on a copper grid covered with a holey carbon film.

\subsection{Electrical characterization}

For the electrical measurements, the electrodes were deposited on the sintered pellets with fired-on silver paste (Johnson Matthey, E1100). Frequency-dependent dielectric permittivity and loss were measured using an Agilent 4294 precision impedance analyser. The temperature dependence of dielectric permittivity and loss was measured from $25^{\circ} \mathrm{C}$ to $450{ }^{\circ} \mathrm{C}$, with a heating rate of $5^{\circ} \mathrm{C} \mathrm{min}-1$, at selected frequencies $(50 \mathrm{kHz}, 100 \mathrm{kHz}, 500 \mathrm{kHz}$ and $1 \mathrm{MHz}$ ), using an Agilent 4284A LCR meter connected to a furnace (Lenton, LTF). The P-E hysteresis loops were measured by a ferroelectric hysteresis measurement tester (NPL, UK), at a frequency of $10 \mathrm{~Hz}$ [37].

\section{Results and Discussion}

The XRD patterns of the calcined powders (supporting information Figure S1) indicate that a small amount of $\mathrm{Bi}_{2} \mathrm{Fe}_{4} \mathrm{O}_{9}$ (JCPDS No. 25-0090) is formed as a secondary phase in powders calcined at low temperatures, but is absent at $910{ }^{\circ} \mathrm{C}$ and above. Selected area electron diffraction (SAED) patterns of BSF15 powder calcined at $910{ }^{\circ} \mathrm{C}$ are shown in Figure 1 (a-f). The analysis of the reflection conditions (0kl: $k+I=2 n ; h 0 l: h=2 n ; h 00: h=2 \mathrm{n} ; 0 k 0: k=2 \mathrm{n} ; 00 l: I=$ $2 n$ ) on the SAED patterns points towards the Pnam (No. 62) space group, which differs from the 
previous studies reporting the presence of an AFE structure with space group Pbam (No. 55) [13, 18]. The unit cell based on the Pnam space group has the following relation to the pseudo-cubic perovskite cell: $a=\sqrt{2} a_{\mathrm{c}}, b=2 \sqrt{2} a_{\mathrm{c}}, c=4 a_{\mathrm{c}}$, where $a_{\mathrm{c}}$ is the unit cell parameter of the pseudocubic perovskite, and is illustrated diagrammatically in Figure S2. Thus, the $0 k 0$ reflection corresponds to $1 / 4(h h 0)$ in the cubic sub-cell (Figure 1a), while the $00 /$ reflection in the Pnam space group corresponds to $1 / 4(00 /)$ in the cubic sub-cell (Figure 1c). The appearance of the forbidden reflections $00 /(I \neq 2 n)$ on the [210] and the [110] patterns, as well as $h 00(h \neq 2 n)$ and $0 k 0(k \neq 2 n)$ on the [001] and the [011] patterns, are due to double diffraction; this follows from the fact that the reflections disappear when rotating the crystal away from the perfect orientation around the corresponding axis. Figure $1 \mathrm{f}$ shows the diffraction pattern of a crystal with $60^{\circ}$-rotation twins, oriented along the [401] direction in the structure.

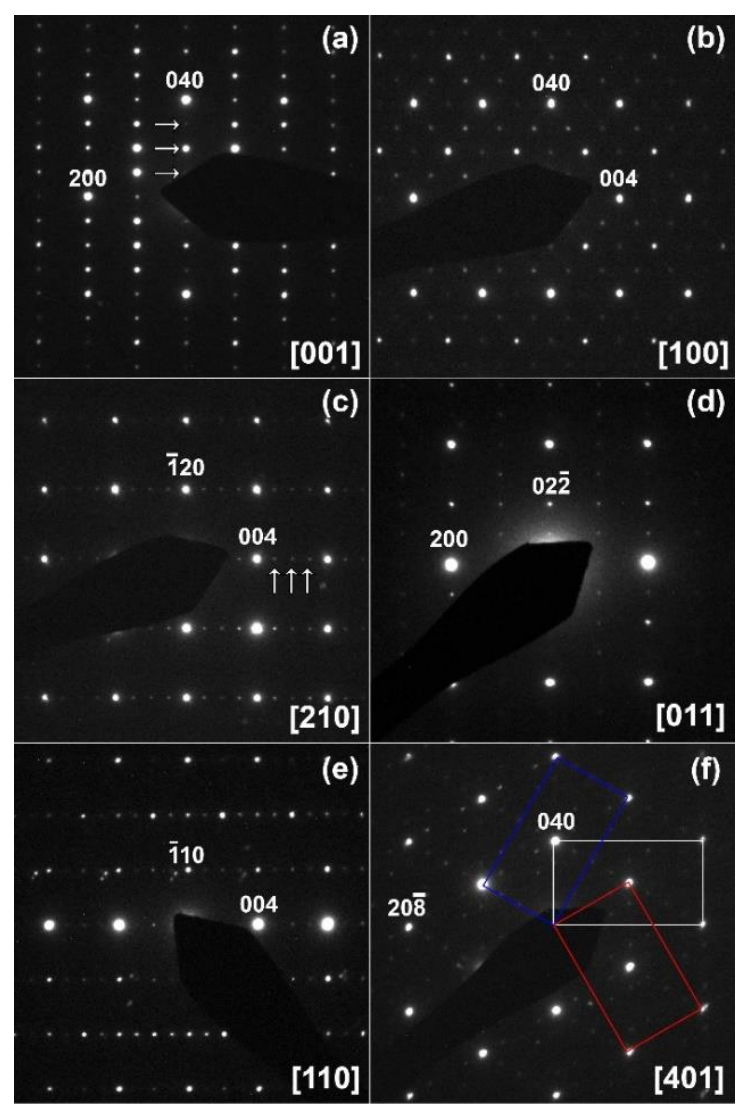


Figure 1. SAED patterns of BSF15 taken along main zone axis (a) [001], (b) [100], (c) [210], (d) [011], (e) [110] and (f) [401] from $60^{\circ}$-rotation twins; white arrows mark $1 / 4(h h 0)$ and $1 / 4(00 /)$ in the cubic sub-cell in patterns (a) and (c), respectively. Red, blue and white rectangles mark unit cells of three rotational twins.

The fitted diffraction profile of the BSF15 powder calcined at $910^{\circ} \mathrm{C}$ is shown in Figure S3. Gil-González et al. reported a Pbam single phase in BSF15 ceramics prepared by mechanochemical synthesis [18], while Levin et al showed that the differences between the Pbam and Pnam structures could not be identified through Rietveld refinement, as both phases produced comparable fitting quality [38]. However, the best fit achieved here was obtained using a three-phase structural model with FE R3c, AFE Pnam and PE Pnma phases. The refined parameters are summarized in Table S1. Figure S4 shows the refined weight fractions of the $R 3 c$, Pnam and Pnma phases as a function of the calcination temperature for BSF15 powder. The weight fractions confirm that the calcination conditions affect the phase separation and homogeneity of the samples, as proposed by Khomchenko et al. [23] and Walker et al. [39]. The weight fraction of the $R 3 c$ phase generally decreases, while that of the Pnam phase increases with increasing calcination temperature. The weight fraction of the Pnma phase does not show significant variations over the calcination temperature range studied. It is suggested that the Pnma phase corresponds to a samarium-rich phase with a similar structure to that of $\mathrm{SmFeO}_{3}$. Indeed, the cell dimensions of this phase (Table S1) are very similar to those of $\mathrm{SmFeO}_{3}[40$ ]. An increase of the calcination temperature is seen to favour the Pnam phase over the $R 3 c$ phase. Since the $R 3 C$ phase is stable within the Sm-deficient side of the morphotropic phase boundary, 
its presence in the samples calcined at lower temperatures suggests that these temperatures are insufficient for significant samarium diffusion. This would result in an inhomogeneous samarium distribution, with samarium-rich areas of the Pnam and/or Pnma symmetry and samarium-deficient regions with the rhombohedral $R 3 c$ symmetry.

In order to achieve both high weight fraction of the Pnam phase and low dielectric loss, the powders $x=0.150,0.160,0.165$ were calcined at $910{ }^{\circ} \mathrm{C}$ and that for $x=0.180$ at $950{ }^{\circ} \mathrm{C}$. The ceramics were sintered at $800{ }^{\circ} \mathrm{C}$ to avoid $\mathrm{Bi}_{2} \mathrm{O}_{3}$ evaporation and achieved relative densities above $95 \%$ in all cases. The XRD patterns of the $\mathrm{Bi}_{1-x} \mathrm{Sm}_{x} \mathrm{FeO}_{3}$ powders and ceramics are shown in Figure S5a and Figure S5b, respectively. All the patterns were fitted using a multi-phase structural model considering the coexistence of the R3c, Pnam and Pnma phases. The variation of the weight fractions of the three phases as a function of $\mathrm{Sm}$ concentration for $\mathrm{Bi}_{1-x} \mathrm{Sm}_{x} \mathrm{FeO}_{3}$ powder is shown in Figure $2 a$. The weight fraction of the $R 3 c$ phase decreases with increasing Sm concentration and in BSF18 the rhombohedral phase is absent. The fraction of the Pnam phase decreases and that of the Pnma phase increases with increasing Sm concentration. This indicates that in the compositional range studied, Sm substitution favours the formation of the Pnma phase at the expense of the $R 3 c$ and Pnam phases. The variation of the weight fraction in each phase as a function of the Sm substitution level for the ceramics (Figure 2b) showed similar trends to those in powder samples, but with a larger fraction of the Pnma phase and a lower fraction of the Pnam phase. It is suggested that SPS sintering further promotes the formation of the Pnma phase. 
(a)

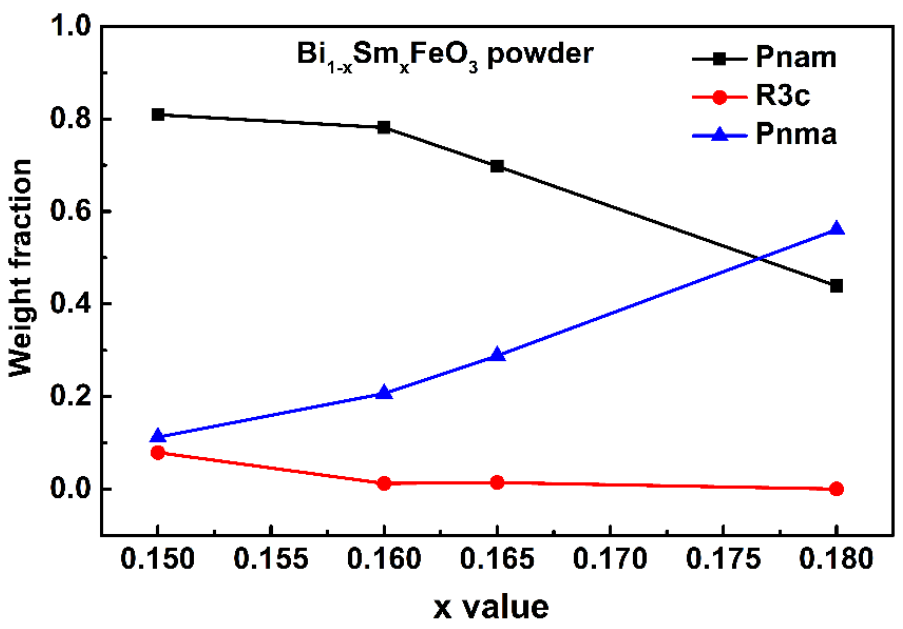

(b)

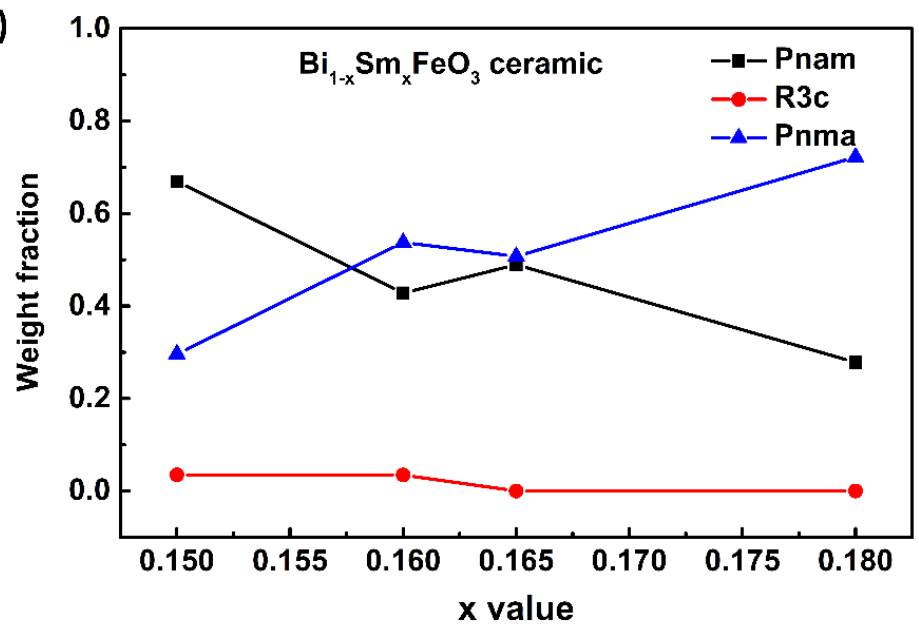

Figure 2. Weight fractions of the $R 3 c$, Pnam and Pnma phases as a function of Sm content in (a) $\mathrm{Bi}_{1-x} \mathrm{Sm}_{x} \mathrm{FeO}_{3}$ powders; (b) $\mathrm{Bi}_{1-x} \mathrm{Sm}_{x} \mathrm{FeO}_{3}$ ceramics $(x=0.150,0.160,0.165$ and 0.180 ). Error bars are smaller than the symbols used.

SEM images of the BSF15 and BSF18 ceramics are shown in Figure 3 as a representative example, with images for other compositions given in the supporting information as Figure S6. The $\mathrm{Bi}_{25} \mathrm{FeO}_{40}$ phase is mainly present at the grain boundaries, while $\mathrm{Bi}_{2} \mathrm{Fe}_{4} \mathrm{O}_{9}$ is evidenced within the grains, in agreement with previous studies [3]. It has been reported that in the 
temperature range $447^{\circ} \mathrm{C}-767^{\circ} \mathrm{C}, \mathrm{Bi}_{25} \mathrm{FeO}_{40}$ is thermodynamically more stable than $\mathrm{BiFeO}_{3}[3$ ]. While the SPS sintering temperature was $800^{\circ} \mathrm{C}$, the post-sintering annealing temperature was lower $\left(700^{\circ} \mathrm{C}\right)$ and may account for the presence of the $\mathrm{Bi}_{25} \mathrm{FeO}_{40}$ secondary phase.
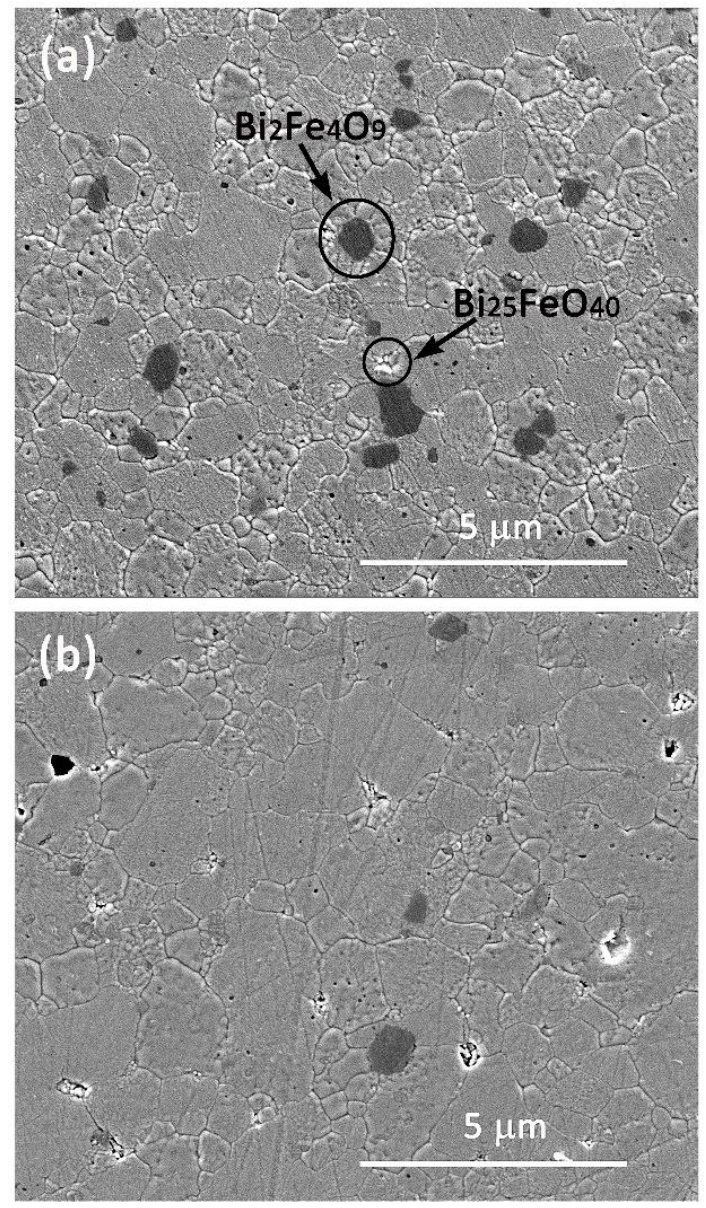

Figure 3 SEM image of (a) BSF15 and (b) BSF18 ceramics. The $\mathrm{Bi}_{2} \mathrm{Fe}_{4} \mathrm{O}_{9}$ (dark grey) and $\mathrm{Bi}_{25} \mathrm{FeO}_{40}$ (bright) phases are highlighted by circles.

Figure 4 shows the frequency dependent dielectric behaviour of the $\mathrm{Bi}_{1-x} \mathrm{Sm}_{x} \mathrm{FeO}_{3}(x=$ $0.150,0.160,0.165$ and 0.180 ) ceramics at room temperature. In general, the measured 
permittivity values $\varepsilon_{r}$ (Figure $4 a$ ) are larger than those of pure $\mathrm{BiFeO}_{3}$ [41]. This can be attributed to the enhanced polarizability of the MPB compositions [42]. The low values of dielectric loss factor $\tan \delta$ (Figure $4 b$ ) suggest a low concentration of oxygen vacancies, i.e. iron likely remains in the $3+$ oxidation state [6]. The permittivity increases with increasing Sm concentration. The dependence of the dielectric loss on the Sm content varies somewhat with frequency and does not show a clear compositional trend at higher frequencies. Nevertheless, the composition with the highest level of Sm-substitution shows the lowest dielectric loss over the entire studied frequency range. Additionally, all the Sm-substituted compositions show significantly lower dielectric loss than unsubstituted $\mathrm{BiFeO}_{3}$, consistent with the reports of Sun et al. [19] and Uchida et al. [43]. For Sm-substituted $\mathrm{BiFeO}_{3}$ thin films, the maximum permittivity has been reported to occur at $x=0.14$ [16]. In the present study, the maximum permittivity of the bulk ceramics is observed at $x=0.180$. 


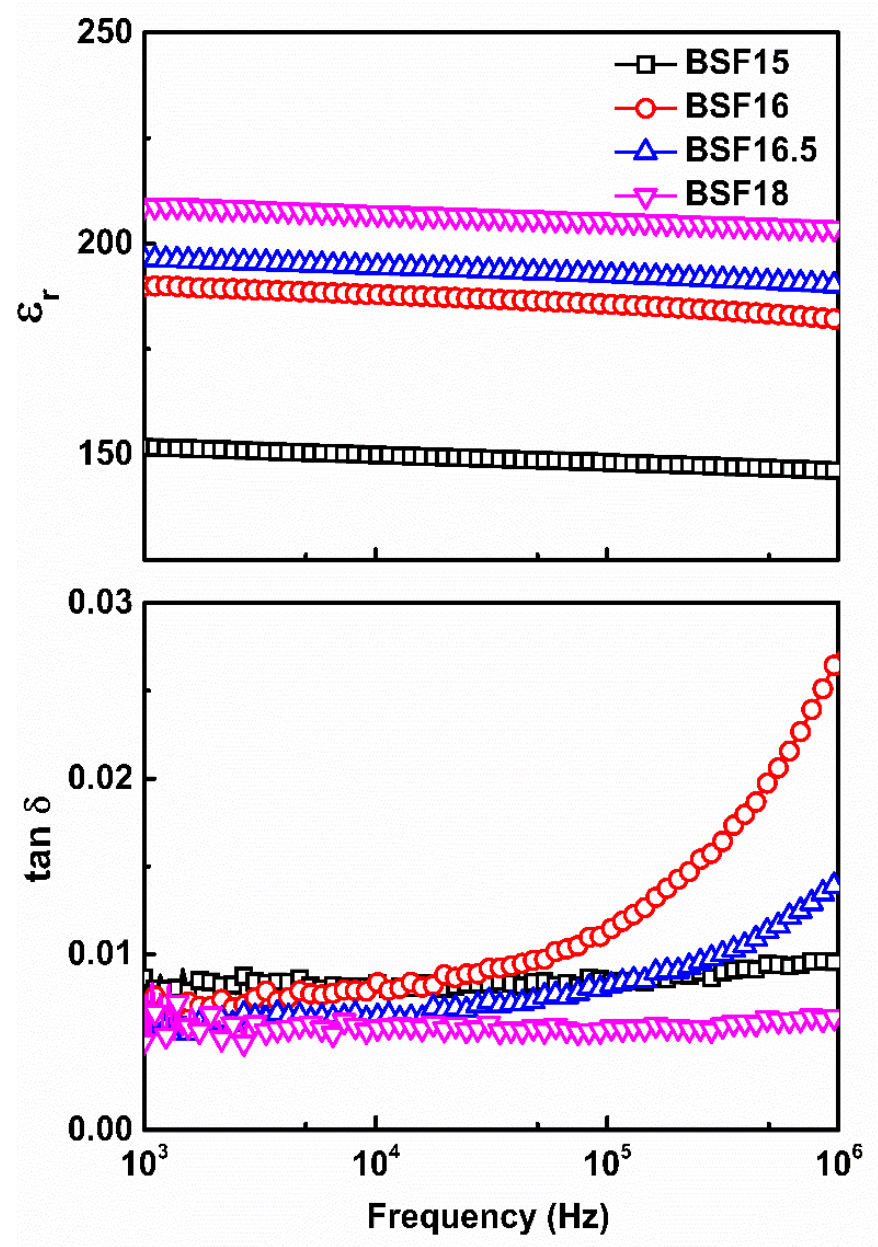

Figure 4. Frequency dependence of (a) relative permittivity $\varepsilon_{r}$ and (b) dielectric loss factor $\tan \delta$ for $\mathrm{Bi}_{1-x} \mathrm{Sm}_{x} \mathrm{FeO}_{3}$ ceramics at room temperature.

Phase transitions in the $\mathrm{Bi}_{1-x} \mathrm{Sm}_{x} \mathrm{FeO}_{3}$ system were investigated by variable temperature XRD. Figure 5 shows detail of the XRD patterns of the BSF15 calcined powder, at selected temperatures over the temperature range $25^{\circ} \mathrm{C}-300^{\circ} \mathrm{C}$, on heating and cooling. The intensity of the peaks of the PE Pnma phase (at about $25.4^{\circ}, 41.2^{\circ}, 47.7^{\circ}$ and $52.8^{\circ} 2 \theta$ ) increases with increasing temperature, while the reflections associated with the AFE Pnam phase (at about $29.2^{\circ}, 45.8^{\circ}, 49.5^{\circ}, 51.6^{\circ}$ and $55.3^{\circ} 2 \theta$ ) show decreasing intensity with increasing temperature. 
For the BSF18 calcined powder (Figure S7), the peaks at about $45.8^{\circ}$ and $51.6^{\circ}$ correspond to the AFE Pnam phase and gradually disappear with increasing temperature.

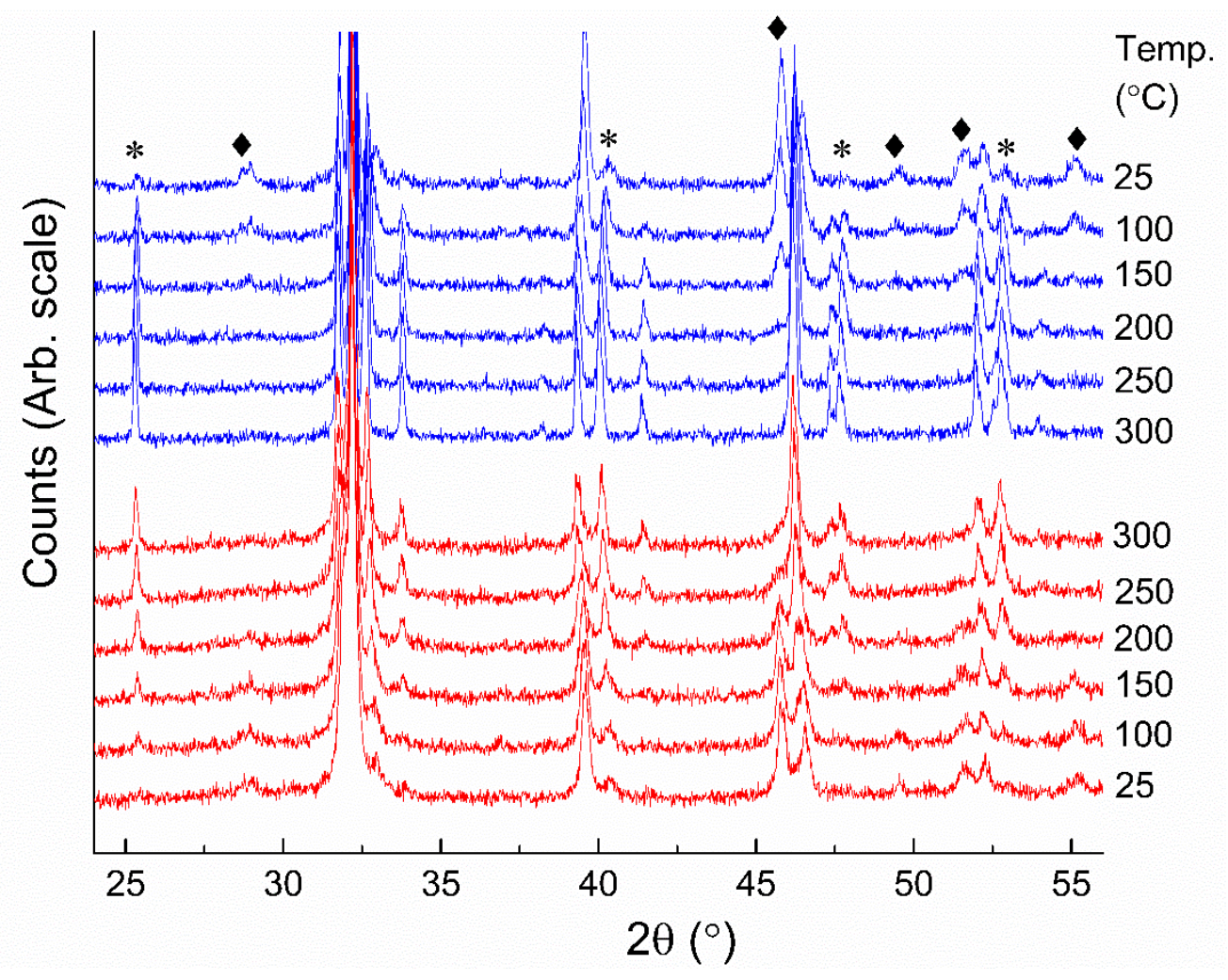

Figure 5. Detail of X-ray diffraction patterns for BSF15 powder (calcined at $910^{\circ} \mathrm{C}$ ) on heating (red) and cooling (blue) at selected temperatures over the range $25^{\circ} \mathrm{C}$ to $300{ }^{\circ} \mathrm{C}$. The peaks belonging exclusively to the Pnma phase are marked by asterisks and those of the Pnam phase are marked by diamonds.

Figure 6a shows the weight fractions of the R3c, Pnam and Pnma phases as functions of temperature for the BSF15 powder. Both the Pnam and $R 3 C$ phases gradually transform to the Pnma phase with increasing temperature, confirming that the Pnma phase is the high- 
temperature stable phase. The Pnam phase completely disappears at $450^{\circ} \mathrm{C}$. Figure $6 \mathrm{~b}$ shows the refined weight fractions of the Pnam and Pnma phases as functions of temperature for the BSF18 powder. The Pnam phase gradually transforms to the Pnma phase upon heating; the transition is completed at about $300{ }^{\circ} \mathrm{C}$, which is lower than the transition temperature for BSF15. The weight fraction of each phase before and after the experiment showed some differences, which are associated with the thermal hysteresis around the phase transition.

(a)

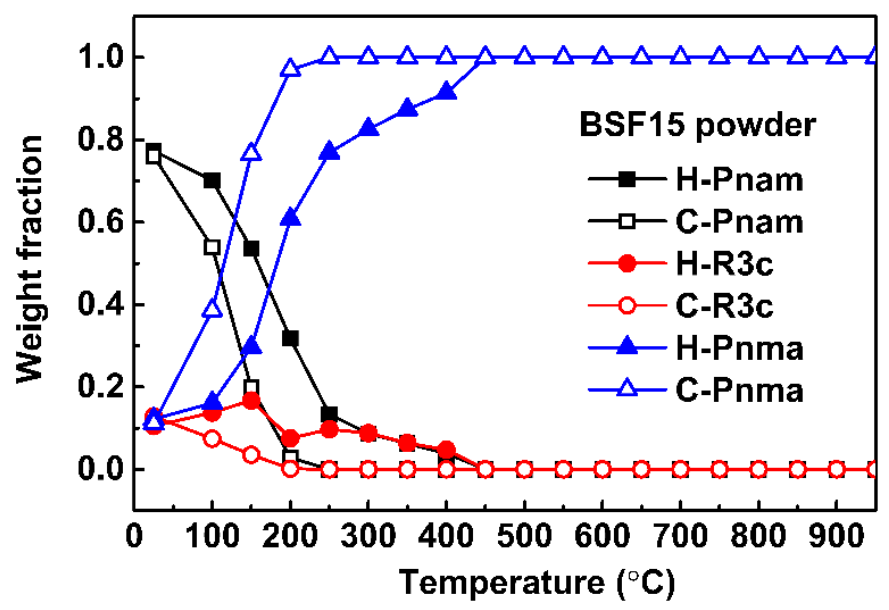

(b)

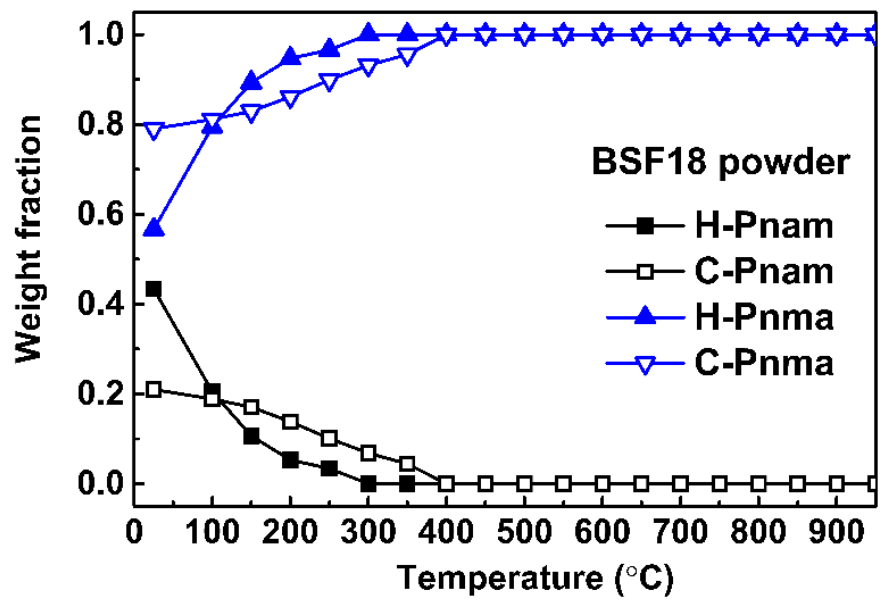

Figure 6. Refined weight fractions of the $R 3 c$, Pnam and Pnma phases as functions of temperature for (a) BSF15 powder and (b) BSF18 powder. 
Figure 7a shows the temperature dependence of dielectric permittivity $\varepsilon_{\mathrm{r}}$ and loss factor $\tan \delta$ of the BSF15 ceramic during heating. At around $200{ }^{\circ} \mathrm{C}$, the permittivity shows a peak accompanied by a minimum in dielectric loss, which correlates with the temperature where the highest rate of decrease in the Pnam phase content was observed in the X-ray data (Figure 6a). Thus, the peak at $200{ }^{\circ} \mathrm{C}$ can be attributed to the transition from the AFE Pnam phase to the PE Pnma phase. This is mostly likely the same event identified by Gil-González and co-workers at $175{ }^{\circ} \mathrm{C}$ by differential scanning calorimetry and X-ray diffraction, which they attributed to a Pbam-Pnma transition [18]. As the samarium content increases, the permittivity peak shifts to lower temperatures (the thermal dependencies of dielectric behaviour for BSF16 and BSF16.5 ceramics are shown in Figures S8a and S8b, respectively). In BSF18, this peak is observed at 100 ${ }^{\circ} \mathrm{C}$ (Figure 7b), where the largest drop in the Pnam phase fraction was detected (Figure 6b). This temperature is close to the phase transition temperature detected in BSF17.5 by Gil-González et al. at $150{ }^{\circ} \mathrm{C}$ and attributed to the Pbam+Pnma/Pnma transformation [18]. The stabilization of the Pnma phase might be attributed to the effect of the substitution of $\mathrm{Bi}^{3+}$ ions by smaller Sm ${ }^{3+}$ ions ( $r=1.36 \AA$ and $1.28 \AA$, respectively for the ions in 12 coordinate geometry [44]), resulting in a lattice distortion and unit cell volume reduction $[45,46]$. The peak at about $400{ }^{\circ} \mathrm{C}$, visible in the high frequency curves in all compositions, is probably related to the antiferromagnetic Néel temperature, in agreement with Gil-González et al. [18]. 

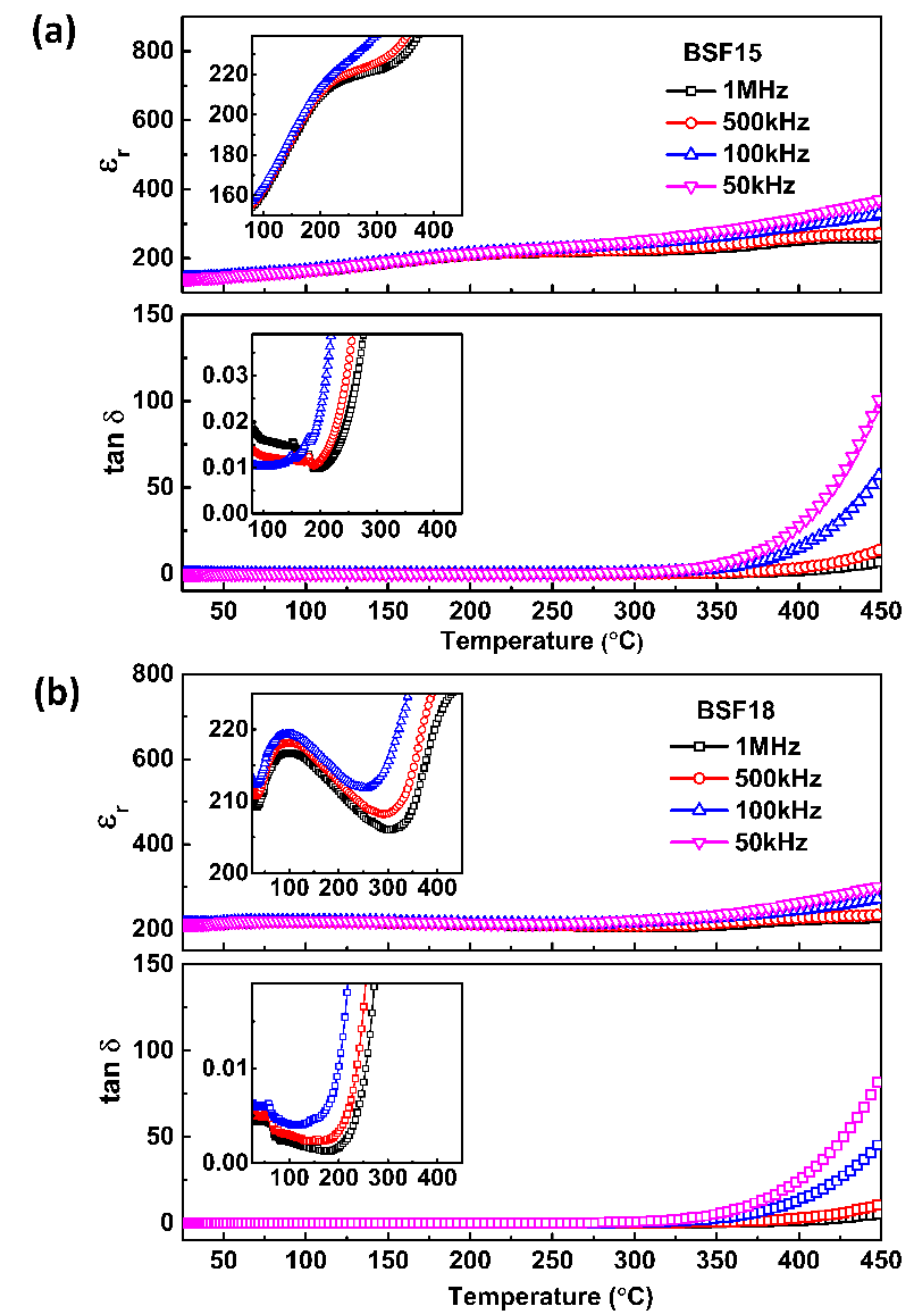

Figure 7. Temperature dependence of relative permittivity $\varepsilon_{r}$ and dielectric loss factor tan $\delta$ at selected frequencies for (a) BSF15 and (b) BSF18 ceramics on heating, with detail shown inset.

The room temperature current-electric field (I-E) and the polarization-electric field (P-E) hysteresis loops of the BSF15 and BSF18 ceramics are shown in Figure 8 and those of the BSF16 and BSF16.5 ceramics are shown in Figure S9. According to the I-E curves, the electrical conductivity of Sm-substituted ceramics is significantly lower than that of pure $\mathrm{BiFeO}_{3}$ (see Ref.1). The current peak (labelled " $A$ ") observed in the I-E loop of BSF15 is indicative of domain 
switching in the FE phase, which is consistent with the presence of the $R 3 c$ structure at room temperature (Figure 2). The lower P-E loop hysteresis and the suppression of the peak in the I-E loops in ceramics with higher Sm content indicates the reduction of the electrical conductivity and the suppression of FE behaviour. Additionally, the breakdown strength of the ceramics has been greatly improved in compositions with higher Sm content, compared to those previously reported $[27,47,48]$. However, double P-E hysteresis loops characteristic of reversible AFE $\leftrightarrow$ FE transitions were not observed, despite the presence of the AFE Pnam phase observed in the TEM images (Figure 1).

(a)

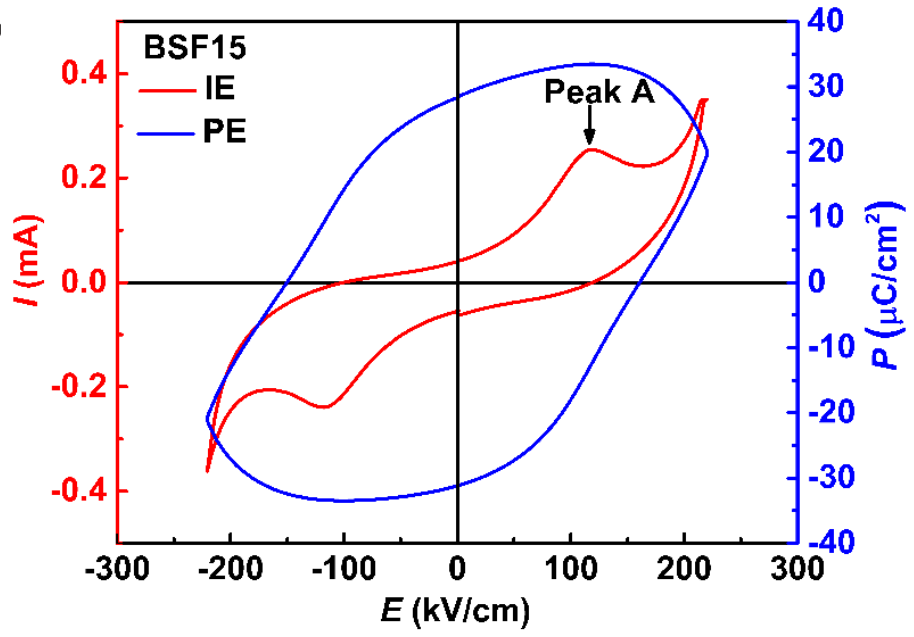

(b)

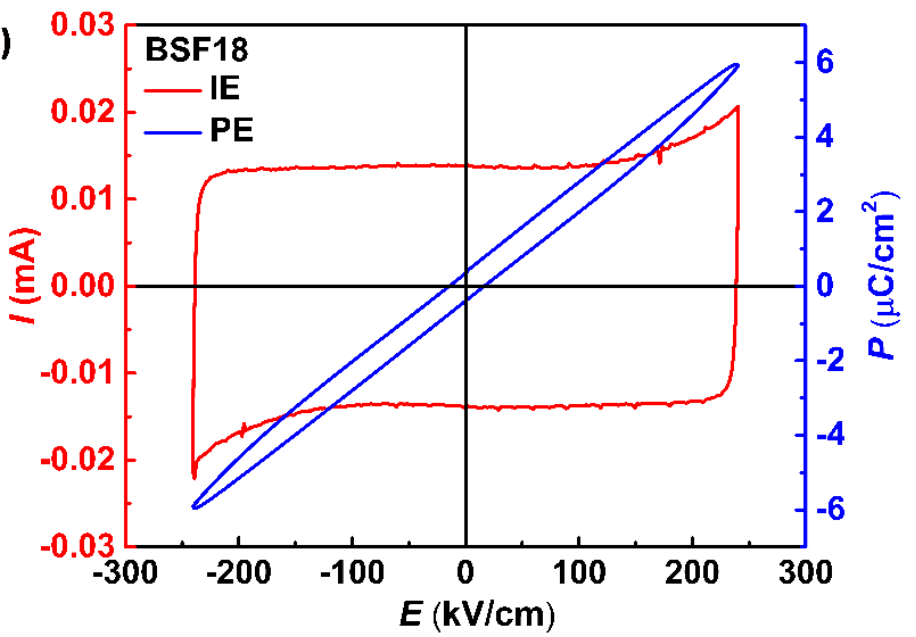


Figure 8. Current-electric field (I-E) and polarization-electric field (P-E) hysteresis loops of (a) BSF15 and (b) BSF18 ceramics.

\section{Conclusions}

Through a detailed diffraction investigation, the existence of an antiferroelectric phase with Pnam symmetry in $\mathrm{Bi}_{1-x} \mathrm{Sm}_{x} \mathrm{FeO}_{3}$ ceramics, with compositions close to the morphotropic phase boundary $(0.15 \leq x \leq 0.18)$ has been confirmed. The antiferroelectric Pnam phase coexists with the ferroelectric $R 3 c$ and paraelectric Pnma phases, whose relative fractions vary with the level of Sm substitution and the calcination conditions. In particular, the fractions of both the $R 3 c$ phase and the Pnam phases decrease with increasing Sm concentration. The fraction of the Pnam phase increases and that of the $R 3 c$ phase decreases with increasing calcination temperature. The fractions of $R 3 c$ and the Pnam phases progressively reduce with increasing temperature in favour of the Pnma phase, with a rate that strongly depends on the Sm concentration. Sm-substitution increases the dielectric permittivity, reduces electrical conductivity, increases breakdown strength and suppresses ferroelectric switching. The results indicate that it is possible to tailor phase composition and the consequent dielectric properties through careful selection of composition and calcination conditions. 


\section{Acknowledgements:}

Chuying Yu acknowledges a scholarship from the China Scholarship Council. Dou Zhang and Haixue Yan thank the Royal Academy of Engineering for a UK and China travel grant. Vladimir Koval acknowledges the Grant Agency of the Slovak Academy of Sciences (Grant No. 2/0059/17)

\section{References}

[1] G. Catalan, J.F. Scott, Physics and applications of bismuth ferrite, Adv. Mater. 21(24) (2009) 2463-2485.

[2] H. Kuo, Y. Shu, H. Chen, C. Hsueh, C. Wang, Y. Chu, Investigation of nanodomain pattern and piezoelectric behavior of mixed phases in epitaxial $\mathrm{BiFeO}_{3}$ films, J. Eur. Ceram. Soc. 31(16) (2011) 3063-3071.

[3] T. Rojac, A. Bencan, B. Malic, G. Tutuncu, J.L. Jones, J.E. Daniels, D. Damjanovic, $\mathrm{BiFeO}_{3}$ ceramics: Processing, electrical, and electromechanical properties, J. Am. Ceram. Soc. 97(7) (2014) 1993-2011.

[4] X. Yan, J. Chen, Y. Qi, J. Cheng, Z. Meng, Hydrothermal synthesis and characterization of multiferroic $\mathrm{Bi}_{1-x} \mathrm{La}_{x} \mathrm{FeO}_{3}$ crystallites, J. Eur. Ceram. Soc. 30(2) (2010) 265-269.

[5] T. Carvalho, P. Tavares, Synthesis and thermodynamic stability of multiferroic $\mathrm{BiFeO}_{3}$, Mater. Lett. 62(24) (2008) 3984-3986.

[6] H. Uchida, R. Ueno, H. Nakaki, H. Funakubo, S. Koda, Ion modification for improvement of insulating and ferroelectric properties of $\mathrm{BiFeO}_{3}$ thin films fabricated by chemical solution deposition, Jpn. J. Appl. Phys. 44(4L) (2005) L561.

[7] V. Koval, I. Skorvanek, M. Reece, L. Mitoseriu, H. Yan, Effect of dysprosium substitution on crystal structure and physical properties of multiferroic $\mathrm{BiFeO}_{3}$ ceramics, J. Eur. Ceram. Soc. 34(3) (2014) 641-651.

[8] S. Fujino, M. Murakami, V. Anbusathaiah, S.-H. Lim, V. Nagarajan, C. Fennie, M. Wuttig, L. Salamanca-Riba, I. Takeuchi, Combinatorial discovery of a lead-free morphotropic phase boundary in a thin-film piezoelectric perovskite, Appl. Phys. Lett. 92(20) (2008) 202904.

[9] J. Walker, H. Ursic, A. Bencan, B. Malic, H. Simons, I. Reaney, G. Viola, V. Nagarajan, T. Rojac, Temperature dependent piezoelectric response and strain-electric-field hysteresis of rare-earth modified bismuth ferrite ceramics, J. Mater. Chem. C 4(33) (2016) 78597868.

[10] C.-J. Cheng, D. Kan, V. Anbusathaiah, I. Takeuchi, V. Nagarajan, Microstructureelectromechanical property correlations in rare-earth-substituted $\mathrm{BiFeO}_{3}$ epitaxial thin films at morphotropic phase boundaries, Appl. Phys. Lett. 97(21) (2010) 212905. 
[11] D. Damjanovic, Materials for high temperature piezoelectric transducers, Curr. Opin. Solid State Mater. Sci. 3(5) (1998) 469-473.

[12] I. Troyanchuk, M. Bushinsky, D. Karpinsky, O. Mantytskaya, V. Fedotova, O. Prochnenko, Structural transformations and magnetic properties of $\mathrm{Bi}_{1-x} \mathrm{Ln}_{x} \mathrm{FeO}_{3}(\mathrm{Ln}=\mathrm{La}$, Nd, Eu) multiferroics, physica status solidi (b) 246(8) (2009) 1901-1907.

[13] S. Karimi, I. Reaney, Y. Han, J. Pokorny, I. Sterianou, Crystal chemistry and domain structure of rare-earth doped $\mathrm{BiFeO}_{3}$ ceramics, J. Mater. Sci. 44(19) (2009) 5102-5112.

[14] Z. Gabbasova, M. Kuz'min, A. Zvezdin, I. Dubenko, V. Murashov, D. Rakov, I. Krynetsky, $\mathrm{Bi}_{1-} \mathrm{R}_{\mathrm{x}} \mathrm{FeO}_{3}(\mathrm{R}=$ rare earth): a family of novel magnetoelectrics, Phys. Lett. A 158(9) (1991) 491-498.

[15] D. Arnold, Composition-driven structural phase transitions in rare-earth-doped $\mathrm{BiFeO}_{3}$ ceramics: a review, Ultrasonics, Ferroelectrics, and Frequency Control, IEEE Transactions on 62(1) (2015) 62-82.

[16] D. Kan, C.-J. Cheng, V. Nagarajan, I. Takeuchi, Composition and temperatureinduced structural evolution in $\mathrm{La}, \mathrm{Sm}$, and $\mathrm{Dy}$ substituted $\mathrm{BiFeO}_{3}$ epitaxial thin films at morphotropic phase boundaries, J. Appl. Phys. 110(1) (2011) 014106.

[17] D.A. Rusakov, A.M. Abakumov, K. Yamaura, A.A. Belik, G. Van Tendeloo, E. Takayama-Muromachi, Structural evolution of the $\mathrm{BiFeO}_{3}-\mathrm{LaFeO}_{3}$ system, Chem. Mater. 23(2) (2010) 285-292.

[18] E. Gil-González, A. Perejón, P.E. Sánchez-Jiménez, M.A. Hayward, J.M. Criado, M.J. Sayagués, L.A. Pérez-Maqueda, Characterization of mechanosynthesized $\mathrm{Bi}_{1-x} \mathrm{Sm}_{x} \mathrm{FeO}_{3}$ samples unencumbered by secondary phases or compositional inhomogeneity, J. Alloys Compd. 711 (2017) 541-551.

[19] W. Sun, J.-F. Li, Q. Yu, L.-Q. Cheng, Phase transition and piezoelectricity of sol-gelprocessed $\mathrm{Sm}$-doped $\mathrm{BiFeO}_{3}$ thin films on $\mathrm{Pt}(111) / \mathrm{Ti} / \mathrm{SiO}_{2} / \mathrm{Si}$ substrates, J. Mater. Chem. C 3(9) (2015) 2115-2122.

[20] C.-J. Cheng, D. Kan, S.-H. Lim, W. McKenzie, P. Munroe, L. Salamanca-Riba, R. Withers, I. Takeuchi, V. Nagarajan, Structural transitions and complex domain structures across a ferroelectric-to-antiferroelectric phase boundary in epitaxial $\mathrm{Sm}$-doped $\mathrm{BiFeO}_{3}$ thin films, Phys. Rev. B 80(1) (2009) 014109.

[21] D. Kan, L. Pálová, V. Anbusathaiah, C.J. Cheng, S. Fujino, V. Nagarajan, K.M. Rabe, I. Takeuchi, Universal behavior and electric-field-induced structural transition in rareearth-substituted $\mathrm{BiFeO}_{3}$, Adv. Funct. Mater. 20(7) (2010) 1108-1115.

[22] D. Kan, C.J. Long, C. Steinmetz, S.E. Lofland, I. Takeuchi, Combinatorial search of structural transitions: Systematic investigation of morphotropic phase boundaries in chemically substituted $\mathrm{BiFeO}_{3}$, J. Mater. Res. 27(21) (2012) 2691-2704.

[23] V. Khomchenko, J. Paixão, B. Costa, D. Karpinsky, A. Kholkin, I. Troyanchuk, V. Shvartsman, P. Borisov, W. Kleemann, Structural, ferroelectric and magnetic properties of $\mathrm{Bi}_{0.85} \mathrm{Sm}_{0.15} \mathrm{FeO}_{3}$ perovskite, Cryst. Res. Technol. 46(3) (2011) 238-242.

[24] Z.X. Cheng, A.H. Li, X.L. Wang, S.X. Dou, K. Ozawa, H. Kimura, S.J. Zhang, T.R. Shrout, Structure, ferroelectric properties, and magnetic properties of the La-doped bismuth ferrite, J. Appl. Phys. 103(7) (2008) 07 E507. 
[25] G. Yuan, S. Or, J. Liu, Z. Liu, Structural transformation and ferroelectromagnetic behavior in single-phase $\mathrm{Bi}_{1-x} \mathrm{Nd}_{x} \mathrm{FeO}_{3}$ multiferroic ceramics, Appl. Phys. Lett. 89(5) (2006) $1-3$.

[26] K. Nalwa, A. Garg, A. Upadhyaya, Effect of samarium doping on the properties of solid-state synthesized multiferroic bismuth ferrite, Mater. Lett. 62(6) (2008) 878-881.

[27] K. Kalantari, I. Sterianou, S. Karimi, M.C. Ferrarelli, S. Miao, D.C. Sinclair, I.M. Reaney, Ti-Doping to Reduce Conductivity in $\mathrm{Bi}_{0.85} \mathrm{Nd}_{0.15} \mathrm{FeO}_{3}$ Ceramics, Adv. Funct. Mater. 21(19) (2011) 3737-3743.

[28] V. Khomchenko, J. Paixao, V. Shvartsman, P. Borisov, W. Kleemann, D. Karpinsky, A. Kholkin, Effect of Sm substitution on ferroelectric and magnetic properties of $\mathrm{BiFeO}_{3}$, Scripta Mater. 62(5) (2010) 238-241.

[29] I.O. Troyanchuk, D.V. Karpinsky, M.V. Bushinsky, O.S. Mantytskaya, N.V. Tereshko, V.N. Shut, Phase transitions, magnetic and piezoelectric properties of rare-earthsubstituted $\mathrm{BiFeO}_{3}$ ceramics, J. Am. Ceram. Soc. 94(12) (2011) 4502-4506.

[30] C.-J. Cheng, A.Y. Borisevich, D. Kan, I. Takeuchi, V. Nagarajan, Nanoscale structural and chemical properties of antipolar clusters in $\mathrm{Sm}$-doped $\mathrm{BiFeO}_{3}$ ferroelectric epitaxial thin films, Chem. Mater. 22(8) (2010) 2588-2596.

[31] P. Sharma, S. Satapathy, D. Varshney, P. Gupta, Effect of sintering temperature on structure and multiferroic properties of $\mathrm{Bi}_{0.825} \mathrm{Sm}_{0.175} \mathrm{FeO}_{3}$ ceramics, Mater. Chem. Phys. 162 (2015) 469-476.

[32] H. Yan, H. Ning, Y. Kan, P. Wang, M.J. Reece, Piezoelectric Ceramics with Super High Curie Points, J. Am. Ceram. Soc. 92(10) (2009) 2270-2275.

[33] Q. Jiang, H. Ning, Q. Zhang, M. Cain, M.J. Reece, H. Yan, Active ferroelectricity in nanostructured multiferroic $\mathrm{BiFeO}_{3}$ bulk ceramics, J. Mater. Chem. C 1(36) (2013) 56285631.

[34] A. Larson, R. Von Dreele, Los Alamos National Laboratory Report No. LAUR-86-748, 2004.

[35] V. Khomchenko, I. Troyanchuk, D. Karpinsky, S. Das, V. Amaral, M. Tovar, V. Sikolenko, J. Paixão, Structural transitions and unusual magnetic behavior in Mn-doped $\mathrm{Bi}_{1-x} \mathrm{La}_{x} \mathrm{FeO}_{3}$ perovskites, J. Appl. Phys. 112(8) (2012) 084102.

[36] J.-M. Moreau, C. Michel, R. Gerson, W.J. James, Ferroelectric $\mathrm{BiFeO}_{3} \mathrm{X}$-ray and neutron diffraction study, J. Phys. Chem. Solids 32(6) (1971) 1315-1320.

[37] G. Viola, T. Saunders, X. Wei, K. Chong, H. Luo, M. Reece, H. Yan, Contribution of piezoelectric effect, electrostriction and ferroelectric/ferroelastic switching to strainelectric field response of dielectrics, J. Adv. Dielectr 3(01) (2013) 1350007.

[38] I. Levin, M. Tucker, H. Wu, V. Provenzano, C. Dennis, S. Karimi, T. Comyn, T. Stevenson, R. Smith, I. Reaney, Displacive phase transitions and magnetic structures in Nd-substituted $\mathrm{BiFeO}_{3}$, Chem. Mater. 23(8) (2011) 2166-2175.

[39] J. Walker, H. Ursic, A. Bencan, B. Malic, H. Simons, I. Reaney, G. Viola, V. Nagarajan, T. Rojac, Temperature dependent piezoelectric response and strain-electric-field hysteresis of rare-earth modified bismuth ferrite ceramics, J. Mater. Chem. C (2016).

[40] E. Maslen, V. Streltsov, N. Ishizawa, A synchrotron X-ray study of the electron density in $\mathrm{SmFeO}_{3}$, Acta Crystallogr. Sect. B: Struct. Sci. 52(3) (1996) 406-413. 
[41] M.M. Kumar, V. Palkar, K. Srinivas, S. Suryanarayana, Ferroelectricity in a pure $\mathrm{BiFeO}_{3}$ ceramic, Appl. Phys. Lett. 76(19) (2000) 2764-2766.

[42] Y. Yao, C. Zhou, D. Lv, D. Wang, H. Wu, Y. Yang, X. Ren, Large piezoelectricity and dielectric permittivity in $\mathrm{BaTiO}_{3}-\mathrm{xBaSnO}$ system: The role of phase coexisting, EPL (Europhysics Letters) 98(2) (2012) 27008.

[43] H. Uchida, R. Ueno, H. Funakubo, S. Koda, Crystal structure and ferroelectric properties of rare-earth substituted $\mathrm{BiFeO}_{3}$ thin films, J. Appl. Phys. 100(1) (2006) 4106.

[44] Y. Jia, Crystal radii and effective ionic radii of the rare earth ions, J. Solid State Chem. 95(1) (1991) 184-187.

[45] A. Berenov, F. Le Goupil, N. Alford, Effect of ionic radii on the Curie temperature in $\mathrm{Ba}_{1-x-y} \mathrm{Sr}_{x} \mathrm{Ca}_{y} \mathrm{TiO}_{3}$ compounds, Sci. Rep. 6 (2016).

[46] D.Y. Suárez, I.M. Reaney, W.E. Lee, Relation between tolerance factor and Tc in Aurivillius compounds, J. Mater. Res. 16(11) (2001) 3139-3149.

[47] A. Kumar, D. Varshney, Crystal structure refinement of $\mathrm{Bi}_{1-x} \mathrm{Nd}_{x} \mathrm{FeO}_{3}$ multiferroic by the Rietveld method, Ceram. Int. 38(5) (2012) 3935-3942.

[48] X. Chen, Y. Wang, Y. Yang, G. Yuan, J. Yin, Z. Liu, Structure, ferroelectricity and piezoelectricity evolutions of $\mathrm{Bi}_{1-x} \mathrm{Sm}_{x} \mathrm{FeO}_{3}$ at various temperatures, Solid State Commun. 152(6) (2012) 497-500. 